\title{
Tamoxifen and the uterus
}

\section{Women given this drug need careful assessment}

The effects of tamoxifen on the endometrium have caused concern since the late $1980 \mathrm{~s}, 12$ and clear evidence of endometrial changes has now emerged from three studies in postmenopausal women-one case-control study of breast cancer ${ }^{3}$ and two randomised controlled trials, one in women with early breast cancer ${ }^{4}$ and one in healthy women with a family history of the disease. ${ }^{5}$ In most women treated with tamoxifen the endometrium remains atrophic, but epithelial metaplasia, simple hyperplasia, atypical hyperplasia, endometrial polyps, and endometrial cancers have all been reported in women taking a dose of 20 mg/day. ${ }^{6}$ There is evidence that local expression of insulinlike growth factor I and insulin-like growth factor binding protein-3 are important in tamoxifen's uterotrophic effect. ${ }^{7}$

In patients with breast cancer the risk of endometrial changes is justified by the protective effect of tamoxifen against the cancer and against osteoporosis and cardiovascular disease. 8 The risk-benefit equations are, however, different in healthy postmenopausal women taking tamoxifen for as yet unestablished benefits, and in such women pelvic assessment should not be confined to those who have abnormal vaginal bleeding.

No data exist on the incidence of asymptomatic endometrial cancers related to treatment with tamoxifen. So far the abnormal endometrial findings in patients taking tamoxifen have been mostly benign processes, often with an unknown malignant potential. The high rates of asymptomatic precancerous lesions of the endometrium being reported are difficult to reconcile with the small numbers of symptomatic endometrial cancers possibly associated with tamoxifen-estimated at a maximum of 20 per 10000 women per year. ${ }^{4}$ This discrepancy makes any assessment of the usefulness of screening difficult.

Nevertheless, the greater part of the endometrial hazards associated with tamoxifen ought to be preventable. No group of women taking tamoxifen has been clearly identified as being at greater risk than the remainder, but the accumulated dose may be important. 356910 Neither is there an established method of selecting asymptomatic women taking tamoxifen for procedures to exclude preneoplastic or neoplastic uterine lesions. Transvaginal ultrasonography is widely available to measure endometrial thickness and is easy to perform, but it is not very reliable. A finding of a thin rectilinear endometrium ( $<5 \mathrm{~mm}$ ) excludes clinically important endometrial lesions, but a small polyp or a focus of atypical hyperplasia may be missed. A finding of a thickened endometrium may lead to overtreatment and mismanagement.

The sonographic appearance of the endometrium in patients taking tamoxifen may be misleading, because tamoxifen exerts an echogenic and sonolucent effect on the endometrial stroma and myometrium without necessarily causing epithelial disease. Even if an endometrial biopsy and a progesterone challenge test yield negative results repeat procedures are likely to be recommended and formal dilatation and curettage or hysteroscopy and biopsy may still be necessary. Furthermore, transvaginal ultrasonography does not differentiate between benign and malignant lesions, and such lesions may require therapeutic curettage, resection, and even hysterectomy rather than treatment with progestins. Overall, and especially in women taking tamoxifen, the findings on transvaginal ultrasonography should be interpreted with caution. ${ }^{11-13}$ The combination of transvaginal ultrasonography with colour Doppler flow measurement or contrast ultrasonography with intracavitary fluid instillation is being investigated in women taking tamoxifen. ${ }^{513}$

Hysteroscopy is a more reliable diagnostic method: it allows direct visualisation, and biopsy specimens can be taken of lesions such as hyperplasia, polyps, and endometrial carcinoma. At present few gynaecologists are willing to use hysteroscopy as an outpatient procedure, though the hysteroscopes now available are safe and easy to handle; atraumatic insertion is possible. Routine hysteroscopy is better than ultrasonography at ruling out endometrial lesions in healthy asymptomatic women taking tamoxifen.

How often should screening be done? Women with a high risk of breast cancer may also have a risk of endometrial lesions, and their endometrium may overreact to stimulation with tamoxifen. Screening before tamoxifen is started makes sense for perimenopausal and postmenopausal women with breast cancer at high risk of endometrial lesions (those with a late menopause, a history of benign endometrial disorders and certain kinds of cancer, earlier or current use of hormones, obesity, diabetes and hypertension, a history of infertility, and chronic anovulation). If no baseline assessment is made such lesions may grow or later be attributed to tamoxifen. There are no consistent data on the effect of duration of intake and cumulative dose of tamoxifen or on whether the risk is increased after tamoxifen is stopped. Some cases of 
endometrial cancer were detected many months after tamoxifen was stopped, but whether this reflects a persistent risk is unknown.

As in women with a uterus taking oestrogen replacement therapy, a balance with progestins may eliminate or protect against overstimulation by tamoxifen. ${ }^{14} 15$ No data are available on this: polyps have been described in women taking combined treatment, ${ }^{16}$ and tamoxifen remains in some ways a mystery drug with effects on the uterus never seen with oestrogen replacement therapy. The effect of progestins on serum lipoproteins and hence on cardiovascular disease and on the breast are of concern. Certainly some clinicians will argue that not every woman taking tamoxifen should be given a progestin.

Postmenopausal women taking tamoxifen who have not had a hysterectomy should be informed about potential endometrial hazards. Abnormal vaginal bleeding needs prompt endometrial assessment. Many questions remain about endometrial surveillance in healthy women taking

1 Fornander T, Rutqvist IE, Cedermark B, Glas U, Mattsson A, Silfversward C, et al. Adjuvant tamoxifen in early breast cancer: occurrence of new primary cancers. Lancet 1989;i: 117-20.

Neven P, De Muylder X, Van Belle Y, Vanderick G, De Muylder E. Tamoxifen and the uterus and endometrium. Lancet 1989;i:375.

3 Van Leeuwen FB, Benraadt J, Coebergh JWW, Kiemeney LALM, Gimbrere CHF, Otter R, et al. Risk of endometrial cancer after tamoxifen treatment of breast cancer. Lancet 1994; 343:448-52.

4 Fisher B, Constantino JP, Redmond CK, Fisher ER, Wickerham DL, Cronin WH, et al Endometrial cancer in tamoxifen-treated breast cancer patients: findings from the NSABP B-14. F Natl Cancer Inst 1994;86:527-37.

5 Kedar RP, Bourne TH, Powles TJ, Collins WP, Ashley SE, Cosgrove DO, et al. Effects of tamoxifen on the uterus and ovaries in a randomised breast cancer prevention trial. Lancet tamoxifen on the us

6 Ismail SM. Pathology of endometrium treated with tamoxifen. 7 Clin Pathol 1994;47:827-33.

7 Huynh H, Pollak M. Uterotrophic actions of estradiol and tamoxifen are associated with inhibition of uterine insulin-like growth factor binding protein 3 gene expression. Cancer Res 1994;54:3115-9. the drug. Screening is feasible, and several approaches need evaluation. The benefits of screening and of other approaches to prevention have yet to be defined. Many of the questions may be answered if screening and preventive measures are included in trials of tamoxifen for the prevention of breast cancer.

P NEVEN

Consultant in obstetrics and gynaecology $X$ DE MUYLDER

Consultant in obstetrics and gynaecology

Y VAN BELLE

Consultant in obstetrics and gynaecology

R CAMPO

Consultant in obstetrics and gynaecology

G VANDERICK

Consultant in obstetrics and gynaecology

Department of Obstetrics and Gynaecology,

Algemene Kliniek St-Jan,

B-1000 Brussels,

Belgium

8 Leake RE. Side effects of adjuvant tamoxifen. BMF 1991;303:1061.

9 De Muylder X, Neven P, de Somer M, Van Belle Y, Vanderick G, De Muylder E. Endometrial lesions under tamoxifen. Int $\mathcal{F}$ Gynaecol Obstet 1991;36:127-30.

10 Cohen I, Rosen DJD, Shapira J, Cordoba M, Gilboa S, Altaras MM, et al. Endometrial changes in postmenopausal women treated with tamoxifen for breast cancer. $\mathrm{Br} \mathcal{F}$ Obstet Gynaecol 1993;100:567-70.

11 Hulka CA, Hall DA. Endometrial abnormalities associated with tamoxifen therapy for breast cancer Sonographic and pathologic correlation. Am F Radiol 1993;160:809-12.

12 Cohen I, Rosen DJD, Tepper P, Cordoba M, Shapira Y, Altaras MM, et al. Ultrasonographic evaluation of endometrium and correlation with endometrial sampling in postmenopausal patients treated with tamoxifen. $\mathcal{F}$ Ultrasound Med 1993;5:275-80.

13 Goldstein SR. Unusual ultrasonographic appearance of the uterus in patients receiving tamoxifen. Am $\mathcal{f}$ Obstet Gynaecol 1994;170:447-51.

14 Powles TJ. Tamoxifen and oestrogen replacement. Lancet 1990;338:48.

15 Powes TJ. Tamoxifen and oestrogen replacement. Lancet 1990,338.48.

16 Neven P, De Muylder X, Van Belle Y, Vanderick G, De Muylder E. Hysteroscopic follow-up during tamoxifen treatment. Eur f Obstet Gynecol Reprod Biol 1990;35:235-8.

\title{
Finding a way through the cost and benefit maze
}

\author{
Standardised instruments are needed
}

Two months ago the NHS Executive sent its letter "Improving the effectiveness of the NHS" to all district general managers, chief executives of trusts, and family health services authorities (for distribution to general practice fundholders). ${ }^{12}$ Earlier letters had drawn their attention to the NHS research and development programme and existing evidence on clinical effectiveness ${ }^{3}$ and asked them to purchase less during 1995-6 of two or more procedures known to be ineffective and more of at least two effective ones. ${ }^{4}$ The most recent letter outlines the close links between this process and the new NHS research and development strategy. In its turn, the requirement to identify effective procedures should focus an intense spotlight on available research findings and have profound implications for future NHS research, especially since effectiveness covers "costs, outcomes, and acceptability to patients and society."5

It is salutary to examine the degree to which current research findings can provide this information for interventions used after myocardial infarctions. Coronary heart disease is the most common cause of death in Britain, consuming an estimated $2.5 \%$ of NHS resources and costing nearly $£ 10$ billion a year in lost production and hospital care. A comprehensive review of recent advances in cardiology shows the difficulties decision makers (in both purchaser and provider organisations) will encounter when interpreting research findings in this discipline ( $p$ 1343). ${ }^{6}$
Interventions after myocardial infarction are likely to interest decision makers because the number of options has increased greatly in recent years, especially with the development of thrombolytic treatment, and this technological explosion is set to continue. When streptokinase and tissue plasminogen activator are taken as examples of thrombolytic agents already evaluated, the literature shows that far greater effort has been expended in large clinical trials than in identifying which outcomes (other than death) should be measured and how different outcomes might be valued to measure overall benefit. ${ }^{6}$ In the case of tissue plasminogen activator, for example, increases in adverse effects, such as stroke, have to be balanced against fewer deaths. Although these might be treated in an additive manner-for example, reduction in deaths minus increase in stroke ${ }^{5}$ - quality of life should ideally also enter the equation. To date, extremely few trials have incorporated measures of the quality of life, perhaps because they cannot be obtained retrospectively from medical records. ${ }^{7}$

As defined above, effectiveness includes costs as well as outcomes. ${ }^{5}$ This is particularly important in the case of streptokinase and tissue plasminogen activator, where costs differ substantially and measurable differences in benefit are small. ${ }^{6}$ However, the literature contains few studies of cost effectiveness, and those that have been reported show little consistency in methods, costs mea- 